\title{
Éthique procédurale, entre petits arrangements et transgression: comparaison Brésil-France ${ }^{\star}$
}

\author{
Severine Colinet, ${ }^{I, \star \star}$ Izabel Friche Passos ${ }^{I I}$ \\ ${ }^{I}$ Université de Cergy-Pontoise, Cergy-Pontoise, France \\ II Université Fédéral de Minas Gerais, Minas Gerais, Brésil
}

\begin{abstract}
Résumé
Dans une approche socio-historique et à partir de nos propres expériences, cet article se propose de comprendre les enjeux relatifs à l'éthique procédurale en France et au Brésil. Les résultats de cette comparaison montrent la même tension disciplinaire entre sciences sociales et biomédicales. On observe une politique volontariste de régulation de la recherche avec des humains dans les deux pays mais qui en France, contrairement au Brésil, n'est pas encore géneralisée. L'éthique procédurale, caractérisée comme normative, protocolaire et standardisée uniformise la manière de penser les recherches. Confrontés à des logiques antagonistes, les chercheurs sont fortement tentés de contourner les procédures.
\end{abstract}

Mots-clés : Éthique procédurale ; sciences sociales ; Brésil ; France

\section{Procedural ethics, between small arrangements and transgression: comparison Brazil-France}

\begin{abstract}
:
The purpose of this article is to understand and compare the issues related to procedural ethics in France and Brazil, using a socio-historical approach and our experiences. The comparison shows the same disciplinary tension between social and biomedical sciences. In both countries we can see a deliberate policy to regulate the research ethically. In France, contrary to Brazil, the spread of procedural ethics is not completed yet. Procedural ethics characterized by a normative, formal dimension that standardizes the way of thinking, or rather the research. Faced with antagonistic logics, the researchers are strongly tempted to get round the procedures.
\end{abstract}

Keywords: Procedural ethics; social sciences; Brazil; France

\section{Introduction}

Le présent article est issu de nos réflexions en tant que chercheures dans le champ des sciences humaines et sociales (SHS), réflexions relatives à notre récente confrontation aux procédures d'éthique de recherche. Son objectif est de présenter le contexte institutionnel relatif à l'éthique procédurale dans une visée comparative au niveau de sa règlementation en France et au Brésil. La comparaison proposée par cet article est liée à notre participation à un colloque international portant sur le thème de « la recherche avec », c'est-à-dire de la recherche participative, et à notre rencontre lors d'un atelier concernant le questionnement principal suivant : « quand, pourquoi, comment et avec quelles intentions nous arrive-t-il de transgresser les procédures de l'éthique de la recherche $» .^{1}$ Ainsi, cette question nous a incitées à mettre en perspective nos expériences et nos réflexions sur l'éthique procédurale en tentant de dégager les similitudes et les différences relatives à des effets sociétaux de nos deux pays. En effet, l'organisation institutionnelle de l'éthique procédurale dépend, en termes plus généraux, des conceptions politiques de la signification et de

\footnotetext{
^Nos remerciements s'adressent au CNPq/Brésil car cet article a été conçu dans le cadre d'une bourse de Post-doctorat senior reçue par le second auteur.

$\star \star$ Adresse postale : Universidade Federal de Minas Gerais, Faculdade de Filosofia e Ciências Humanas, Departamento de Psicologia. Universidade Federa de Minas Gerais - Pampulha. Belo Horizonte, MG - Brasil. CEP : 31270901. E-mail: severine.colinet@u-cergy.fr, izabelfrichepassos@gmail.com

Il s'agit du Symposium «Faire de la recherche «avec » : enjeux épistémologiques, éthiques, méthodologiques et pratiques en intervention sociale, santé et éducation ", organisé par le Réseau d'échange et de partage international et interdisciplinaire francophone Recherche Avec, à Ottawa/ Canada, du 30 avril au 2 mai 2014.
}

l'importance sociales de la production scientifique. Nous avons mis en perspective les spécificités politico-sociétales relatives à l'éthique procédurale en comparant les systèmes français et brésilien qui présentent des analogies bien que l'on observe des différences importantes dans la mise en application de l'éthique de la recherche en SHS. Bien entendu, il ne s'agit pas de plaquer un modèle sur l'autre, mais bien de mettre en perspective les deux systèmes pour en dégager des similitudes et des spécificités génératrices de connaissances sur un objet qui traverse internationalement les communautés scientifiques, qu'elles relèvent d'ailleurs des sciences sociales ou biomédicales.

Après un état des lieux bibliographique et une présentation de la spécificité de notre recherche, il s'agira d'exposer, dans une approche socio-historique, l'organisation de l'éthique procédurale au Brésil et en France. Puis nous nous attacherons à mettre en perspective les enjeux identifiés de l'éthique procédurale dans ces deux pays, que ce soit au niveau du fonctionnement, des tensions disciplinaires entre sciences sociales et biomédicales ou encore des transgressions pour contourner cette règlementation. En fin d'article, une discussion portera sur le renouvellement de la régulation de l'éthique procédurale, au-delà des comités. La conclusion ouvre sur les perspectives de ce travail.

\section{Etat des lieux bibliographique}

Le thème de l'éthique de recherche n'est pas nouveau. Il est largement exploré dans les champs juridiques, 
médicaux, historiques, sociologiques, philosophiques ou encore théologiques. Les chercheurs, français (LAURENS; NEYRAT, 2010; VASSY; KELLER, 2008; DIET, 2003) brésiliens (GUERRIERO, 2006; MINAYO, 2008; LANGDON, 2008 ; SCHLEMPER JUNIOR; HELLMANN, 2013; GARRAFA; LORENZO, 2013) se sont largement emparés du sujet. Les articles soulignent les limites des procédures éthiques et l'inadéquation de ces procédures aux recherches en sciences sociales et notamment ethnographiques (FASSIN, 2008; DINIZ, 2008).

À notre connaissance, aucune recherche ne porte sur une analyse comparative de l'éthique procédurale en France et au Brésil. Cet article vise donc à combler ce vide bibliographique. Pour mieux comprendre les enjeux relatifs à l'éthique procédurale dans nos deux pays, on se propose d'examiner comparativement le processus d'institutionnalisation, dans une approche socio-historique et à partir de nos expériences de confrontation aux comités d'éthique.

Les deux pays, inscrits sur des continents différents, ont pour point commun d'être influencés par le monde anglo-saxon. Pourtant, l'état d'avancement du processus d'institutionnalisation de l'éthique procédurale en sciences sociales n'est pas le même en France et au Brésil, ce qui permet de mieux comprendre comment cette dynamique d'institutionnalisation se généralise progressivement dans le monde, au point de conduire à une uniformisation des pratiques et donc à une uniformisation du rapport à la recherche ou plutôt aux recherches. Cette uniformisation n'est pas sans conséquences sur la production scientifique et surtout sa diversité.

\section{Approche socio-historique et organisationnelle}

\section{Au Brésil}

Le premier texte régulateur brésilien date de 1996 : Resolução $N^{\circ} 196$ de 10 de outubro de 1996 (BRASIL, 1996). Il s'inscrit dans le contexte de re-démocratisation politique du pays et répond à l'esprit de la Constitution Fédérale de 1988 (BRASIL, 1988). Établi par le Conseil National de Santé, il appartient au Ministère de la Santé, tenant en compte, comme références majeures, les orientations de la Déclaration de Helsinki-Finlande-DH (1964), qui à son tour a été élaborée en 1964 par l'Association Médicale Mondiale, et la Déclaration de Manille (1981), un projet commun de l'Organisation mondiale de la santé et du Conseil des organisations internationales des sciences médicales. Il se réfère aussi aux directives $\mathrm{du}$ Council for International Organizations of Medical Sciences (CIOMS). Comme partout dans le monde, la normalisation brésilienne est totalement orientée vers le code déontologique de la recherche biomédicale. Une dernière révision complémentaire de la résolution N.196/96 - Resolução $N^{\circ}$ 466, de 12 de dezembro de 2012 (BRASIL, 2012) - approuvée en 2012, continue à se référer à la DH jusqu'à sa cinquième révision en 2000 . Le Brésil se positionne de façon contraire à la sixième et dernière révision de la $\mathrm{DH}$, qui a eu lieu en 2008 à Séoul, en Corée, en raison de la flexibilisation du texte sur deux points principaux, à savoir : 1) l'acceptation de l'utili- sation du placebo même quand des traitements efficaces s'appliquent à la maladie en investigation et 2) l'acceptation des différents critères standard de soins de santé selon les pays qui abritent la recherche. Cela signifie que les meilleurs traitements, qui sont considérés comme des références par la recherche, sont disponibles dans le pays où s'effectue la recherche et non les meilleurs déjà disponibles, ce qui fragilise énormément les populations des pays en développement (SCHLEMPER JUNIOR; HELLMANN, 2013; GARRAFA; LORENZO, 2013).

Ces flexibilisations sont dues aux pressions économiques de l'industrie pharmaceutique et de la corporation médicale nord-américaine. À partir de ce moment-là, le Brésil et d'autres pays de l'Amérique Latine commencent à prendre leurs distances par rapport à la Déclaration d'Helsinki, et obéissent à la Carta de Córdoba-Argentine (2008) qui a été proposée pendant le Congrès du Réseau Latino-Américain et de la Caraïbe de Bioéthique, organisé par l'UNESCO et qui réaffirme les principes de la Déclaration Universelle de Bioéthique et des droits de l'Homme approuvés en 2005 par 191 pays du monde entier. Le Brésil innove en introduisant la nécessité de demander aussi aux enfants et adolescents leur consentement à participer à une recherche, dans la mesure de leur compréhension, l'autorisation exclusive de leurs responsables ne suffisant plus (GARRAFA; LORENZO, 2013). La résolution complémentaire 446/12 (BRASIL, 2012) réaffirme les restrictions quant à l'usage de placebo et clarifie les procédures pour garantir le droit des volontaires à la recherche aux soins qui se sont avérés efficaces par la recherche menée.

On doit souligner combien les efforts pour réguler éthiquement la recherche, principalement dans le champ de la santé, sont importants et non seulement pour les pays en développement comme le Brésil. Le gain réel en termes de protection ou de tentatives de protection de la vie humaine est évident. Les normes légales nous aident à faire face à la marchandisation de la santé, dont l'avancée démesurée de l'industrie pharmaceutique est la démonstration la plus flagrante. C'est la seconde industrie la plus lucrative du système capitaliste selon quelques études actuelles (MARTINEZ-HERNAEZ, 2013). La normalisation nous aide aussi à contrôler minimalement la montée sans précédent de la médicalisation.

Maintenant, il y a au Brésil une unique Commission nationale d'éthique de la recherche (CONEP), laquelle est subordonnée au Ministère de la Santé et liée au Conseil National de Santé, dont le code déontologique oriente, ou plutôt normalise, toutes les recherches impliquant des êtres humains, qu'il s'agisse de recherches biomédicales ou de recherches en SHS. À travers le temps, des comités institutionnels appartenant à des organismes locaux de recherche (universitaires, hospitaliers, etc.), appelés Comités d'Éthique dans la Recherche (CEP) et subordonnés à la CONEP, se sont multipliés dans le pays dans la mesure où, selon les déterminations de la CONEP, toutes les recherches faisant appel à des êtres humains doivent être soumises à ce système d'éthique, appelé Système Plateforme Brésil, pour être approuvées. Les CEP sont 
définis comme « des corps collégiaux interdisciplinaires et indépendants, de pertinence publique, consultatifs, délibératifs et éducatifs, créés pour défendre les intérêts des participants de la recherche dans son intégrité et dignité et pour contribuer au développement de la recherche dans les normes éthiques » (BRASIL, 2012, p. 8, notre traduction). En général, tous les comités concernés par une recherche, par exemple le CEP de l'université où elle est basée, le CEP du Secrétariat de santé de l'État, le CEP du Secrétariat de santé de la municipalité... veulent évaluer le même projet de recherche avant de l'autoriser à se mettre en place. Cela signifie plusieurs mois, parfois plus de dix mois d'exhaustives procédures bureaucratiques dupliquées ou triplées.

En ce moment prolifère un débat très polémique sur la centralisation de la normalisation éthique par le Ministère de la Santé. Le problème est que les régulations éthiques envisageant presque exclusivement les questions biomédicales sont totalement insensibles aux questions particulières relatives à la recherche en SHS, principalement pour les disciplines qui présupposent une pratique ou une intervention sociale comme c'est le cas de l'anthropologie, ou de la psychologie sociale et communautaire.

Il existe quelques comités spécifiques pour les SHS, mais ils sont peu nombreux ; le premier a été créé en 2007 à l'Université de Brasilia (SARTI ; DUARTE, 2013). Un exemple intéressant de composition d'un CEP spécifique est fourni par la Faculté de Philosophie et Sciences Humaines de l'Université Fédérale de Bahia qui comprend 12 membres, ses suppléants respectifs étant : un représentant-enseignant de chaque département de la faculté, un représentant-enseignant d'autres départements de l'université, un étudiant diplômé de post-graduation, un représentant des groupes de participants potentiels de recherches (personnes en situation de vulnérabilité, de handicap, etc.), un représentant d'une communauté religieuse (église, congrégation, etc.) et un représentant d'une organisation politique (élu ou militant d'un parti politique).

\section{En France}

La première déclaration internationale sur la recherche impliquant la participation de sujets humains est le Code de Nuremberg (1947), issu du procès des médecins accusés de s'être livrés à des expérimentations cruelles sur des prisonniers et des détenus au cours de la seconde guerre mondiale. Comme pour le Brésil, le développement des comités en France fait suite à la déclaration d'Helsinki (1964) et à celle de Manille (1981). Dans ce pays, les comités d'éthique ont subi l'influence anglo-américaine, notamment à la suite du rapport Belmont (UNITED STATES, 1979) relatif aux « principes éthiques et lignes de conduites pour la protection des sujets humains de la recherche ». Des comités d'éthique se sont développés dans des laboratoires de recherche pour répondre aux exigences des recherches conduites en partenariat avec des équipes anglo-américaines.

La France a été le premier pays à créer un Comité Consultatif National d'Éthique (CCNE) pour les sciences de la vie et de la santé en 1983. En France, les chercheurs en SHS ont été d'abord sollicités à l'occasion de la mise en place de ce CCNE où, pour la première fois, siégeait un collège rassemblant non seulement diverses professions scientifiques, médicales et paramédicales, mais aussi des représentants de la sociologie, de l'anthropologie, de la psychologie, de la philosophie ou du droit. Cette pluridisciplinarité large a soudainement donné une forme de légitimité aux savoirs issus de ces disciplines mais, en même temps, les a identifiés comme des supports à l'énonciation des critères éthiques (HARDY, 2013). Cette visibilité d'un usage possible des SHS en médecine a suscité un intérêt nouveau pour ces chercheurs plutôt ignorés, mais aussi l'espoir d'un soutien bienveillant à l'action médicale dès lors que celle-ci se montre « humaniste ». C'est dans ce contexte que des postes en SHS ont été créés dans les facultés de médecine, afin de doter d'une assise l'enseignement de l'éthique. On constate donc que les sciences sociales se situent entre des opportunités de reconnaissance par les sciences biomédicales et qu'en même temps elles sont dominées. On fait le même constat au Brésil où les chercheurs en SHS jouent un rôle très important (MINAYO, 2008).

La loi No. 88-1138 (FRANCE, 1988), dite loi «HURIET-SÉRUSCLAT », relative à la protection des personnes qui se prêtent à des recherches biomédicales, a institué les Comités Consultatifs de Protection des Personnes se prêtant à des recherches biomédicales (CCPPRB). En parallèle, se sont développés des comités régionaux informels. Les Comités Consultatifs de Protection des Personnes furent remplacés en 2004 par des Comités de protection de personnes (CPP). La loi relative à la politique de santé publique (FRANCE, 2004a) qui visait à mettre en œuvre la législation française en accord avec la Direction Européenne 2001/20/ CE (PARLEMENT EUROPÉEN, 2001), leur a confié un rôle de co-décideur dans l'autorisation des recherches biomédicales. Ils sont donc chargés d'émettre un avis préalable sur les conditions de validité de toute recherche impliquant la personne humaine, au regard des critères définis par l'article L. 1123-7 (FRANCE, 2004b) du Code de la Santé Publique (CSP). La loi Hôpital, patients, santé et territoires No. 2009-879 (FRANCE, 2009) précise que le directeur de l'Agence Régionale de Santé délivre l'autorisation de procéder à des recherches biomédicales. La loi Jardé No. 2012300 (FRANCE, 2012) introduit la notion de « recherche impliquant la personne humaine », notion qui remplace l'expression « recherche biomédicale ». La proposition de loi en 2009 suggérait la soumission de tous les projets de recherche sur la personne au CPP. Cette proposition de loi a eu pour effet la création de comités d'éthique pour les recherches non-interventionnelles au niveau de plusieurs institutions académiques et hospitalières.

Créée en 1978, la CNIL (Commission nationale de l'informatique et des libertés) est obligatoire pour certaines recherches. Elle a pour mission essentielle de protéger la vie privée et les libertés individuelles ou publiques face aux dangers que l'informatique peut faire peser sur les libertés. Le CCITRS (Comité consultatif sur le trai- 
tement de l'information en matière de recherche dans le domaine de la santé) dépendant du Ministère de l'Enseignement Supérieur et de la Recherche est chargé de vérifier les conditions de traitement et de transmission des informations individuelles recueillies dans le cadre d'une recherche biomédicale. Il a pour rôle d'éclairer la CNIL.

Progressivement, des comités d'éthique ont été créés par des organismes de recherche, comme celui du CNRS, (Centre national de la recherche scientifique), de l'INSERM (Institut national de la santé et de la recherche médicale), de l'Institut de recherche pour le développement. À ces comités s'ajoutent les comités de recherche clinique en fonction des spécialités. Même si les sciences sociales sont minoritaires, elles sont toutefois représentées.

Sachant que la composition des comités de protection des personnes est à dominante biomédicale, les universités ont créé des comités d'éthique spécialisés dans les SHS. Il s'agit de comités d'éthique appliquée, d'instances de réflexion, mais ces comités peuvent également avoir un rôle d'évaluation. Ainsi, récemment à la fin des années 2000, des universités (Provence, Descartes, Paris 8 Vincennes-St-Denis) se sont dotées de comités d'éthique. Ces comités sont composés à la fois d'universitaires et de non-universitaires de différentes disciplines. Les Comités des laboratoires, par exemple le Centre de recherche et de documents sur l'Océanie, le laboratoire de psychologie et neurocognition (LPNC), dont l'objectif est la prise en compte de populations spécifiques, vise à répondre aux exigences éditoriales et à anticiper le mouvement d'institutionnalisation des comités d'éthique. En effet, les contrats de recherche européens et maintenant nationaux ont des exigences en matière d'éthique. La Charte européenne des chercheurs stipule d'ailleurs : « les chercheurs doivent adhérer aux pratiques éthiques reconnues et aux principes éthiques fondamentaux de mise dans leur(s) discipline(s), ainsi qu'aux normes éthiques étayées par les différents codes d'éthique nationaux, sectoriels ou institutionnels » (COMMISSION EUROPÉENNE, 2005, p. 14).

Par conséquent, en France, on assiste à un mouvement progressif de généralisation de la soumission aux procédures éthiques qui concernent de plus en plus les recherches en sciences sociales.

Le tableau 1 présente les instances organisationnelles et les principales caractéristiques de l'éthique procédurale au Brésil et en France. Après ce détour socio-historique, les réflexions qui suivent abordent davantage l'éthique procédurale dans l'inscription des pratiques professionnelles des chercheurs.
Tableau 1

Organisation et caractéristiques de l'éthique procédurale au Brésil et en France

\begin{tabular}{|c|c|c|}
\hline & Brésil & France \\
\hline Instances & $\begin{array}{l}\text { CONEP } \\
\text { CEP-Comités d'éthique en recherche } \\
\text { (universités, centres de recherche, } \\
\text { secrétariats de santé régionaux et } \\
\text { municipaux, hôpitaux) }\end{array}$ & $\begin{array}{l}\text { CCNE } \\
\text { CCITRS } \\
\text { CPP } \\
\text { Comités d'éthique (CNRS, INSERM, } \\
\text { Institut de recherche pour le } \\
\text { développement, hôpital, université, } \\
\text { laboratoire) } \\
\text { CNIL }\end{array}$ \\
\hline $\begin{array}{l}\text { Comité } \\
\text { d'éthique de } \\
\text { l'Université }\end{array}$ & Existence systématique & Existence non systématique \\
\hline Référence & Biomédicale & Biomédicale \\
\hline Régulateurs & $\begin{array}{l}\text { Revues médicales, de santé collective } \\
\text { ou en psychologie référencées } \\
\text { Financeurs }\end{array}$ & $\begin{array}{l}\text { Revues médicales ou en psychologie } \\
\text { référencées } \\
\text { Financeurs }\end{array}$ \\
\hline Limites & $\begin{array}{l}\text { Durée de traitement des dossiers } \\
\text { Empilement des dispositifs }\end{array}$ & $\begin{array}{l}\text { Durée de traitement des dossiers } \\
\text { Empilement des dispositifs }\end{array}$ \\
\hline $\begin{array}{l}\text { Principales } \\
\text { caractéristiques }\end{array}$ & Normatif, protocolaire, standardisé & Normatif, protocolaire, standardisé \\
\hline \begin{tabular}{lr}
\multicolumn{2}{|l}{ Positionnement } \\
sociétal par \\
rapport à \\
l'éthique \\
procédurale
\end{tabular} & Obligation depuis 1996 & Mouvement de généralisation en cours \\
\hline
\end{tabular}

\section{L'éthique procédurale : une bureaucratie}

En France comme au Brésil, les doublons relatifs aux instances occasionnent des délais de traitement des dossiers règlementaires très importants. Plus spécifiquement, au Brésil cela pose un problème majeur pour les étudiants de Master qui doivent à la fois obtenir une approbation et dans le même temps ont à peine un an pour terminer leurs recherches. Ainsi, ne serait-ce qu'après une première expérience de confrontation à l'éthique procédurale, les tentations qu'éprouvent les équipes de recherche de transgresser les procédures éthiques règlementaires, dans l'intention d'accélérer la mise en œuvre de la recherche, sont très fortes. En effet, le chercheur se trouve réduit à concilier des logiques antagonistes : la logique procédurale, la logique de la recherche et la logique du financeur. En effet, la logique procédurale implique de ne pas débuter la recherche tant que le protocole de recherche n'est pas validé. Le chercheur entend lancer le protocole de recherche le plus rapidement possible pour pouvoir répondre, par la suite, à l'exigence du financeur en rédigeant un bilan intermédiaire à lui remettre, car ce dernier attend des résultats de recherche et pas la simple description de ce qui a été mis en œuvre pour répondre à la réglementation éthique. La logique du financeur est bien celle d'un retour sur investissement. Il raisonne en se basant sur la date de la signature de la convention, en l'occurrence sur l'année civile, et non pas sur la date réelle du début de la recherche.

Face aux difficultés rencontrées dans la confrontation à l'éthique procédurale, les équipes de recherche développent des tactiques de contournement (KATZ, 2006). Nos expériences de rapport à l'éthique ont mis à jour une ambivalence dans le positionnement des équipes de recherches, entre faire et ne pas faire et entre dire et ne pas dire. 


\section{Faire, ne pas faire}

Cette éthique procédurale qui prend la forme d'une bureaucratie n'est pas sans effet sur la production de connaissances. Des chercheurs anglo-saxons ont déjà montré que les comités d'éthique contribuent à modifier le choix des sujets de recherche ou les méthodes utilisées (VASSY; KELLER, 2008). Elle pousse à penser le dispositif de recherche comme une organisation rationnelle, allant jusqu'à générer une « violence rationnelle » (DIET, 2003 , p. 4). Cette approche pousse les chercheurs à présenter des dispositifs de recherche « classiques » connus par tous et surtout par les sciences biomédicales, sans en expérimenter d'autres. En effet, la volonté d'enrichir le dispositif par la mise en place de nouveaux outils méthodologiques, par exemple, peut être freiné par la nécessité de reconstituer tous les dossiers règlementaires, avec pour effet de différer le projet en moyenne de 6 mois.

\section{Dire sans dire}

Les formulaires des comités obligent le chercheur à détailler techniquement le recueil, le stockage, le codage, le système de sécurité informatique utilisé, ou encore la destruction des données. Dans le même temps, certaines expressions sous-jacentes de conceptions épistémologiques en sciences sociales sont à éviter car méconnues par les sciences biomédicales qui imposent leur vocabulaire. À titre d'exemple, on ne parle pas de sujets, mais de patients, de même, certains termes relatifs à « l'interventionnel » sont à gommer car ne correspondant pas aux normes de la communication dominante. Il existerait donc une rhétorique de l'éthique procédurale normée en fonction des sciences biomédicales. Dans la logique opératoire de la procédure, il existe une « réduction utilitariste du langage à un instrument opératoire » (DIET, 2003, p. 9). La reconnaissance des sciences sociales, de ses spécificités épistémologiques et par conséquent terminologiques fait donc problème dans la mise en application de l'éthique procédurale.

\section{Résistance et transgression : l'exemple de l'anthropologie}

Au Brésil, l'exemple le plus extréme et important de transgression des procédures éthiques formelles nous a été donné par l'Anthropologie. Les anthropologues brésiliens refusent la régulation officielle de l'éthique de la recherche. Ils se sont insurgés contre la Commission Nationale d'Éthique dans la Recherche (CONEP), parce que cette instance officielle de régulation, malgré sa prétention de réguler toutes les recherches impliquant des êtres humains, est tout de même strictement liée au champ de la santé, subordonnée au Ministère de la Santé. En toute rigueur, il n'existe pas de régulation institutionnalisée de la recherche sociale et anthropologique au Brésil. Ce qui ne veut pas dire qu'il n'y a pas un souci éthique dans ces types de recherche, bien au contraire, le problème éthique se pose au chercheurs en sciences sociales comme intrinsèque à la recherche elle-même et est évidemment régulé par la communauté scientifique des égaux.
Une approche comparative signale une contradiction : alors qu'aux Etats-Unis, les premiers codes éthiques de l'anthropologie ont été rédigés à la fin de la seconde guerre mondiale (FASSIN, 2008), la France s'est située à l'écart de cette dynamique, refusant la formalisation de règles (ASSOCIATION FRANÇAISE DES ANTHROPOLOGUES, 1993).

À partir des débats déclenchés principalement par l'Associação Brasileira de Antropologia (ABA) avec d'autres associations adhérentes de sociologie, de psychologie et de service social spécialement, il a été créé, l'année 2013, un Groupe de Travail dans la CONEP ayant pour but d'élaborer une « résolution complémentaire » à joindre à la dernière résolution approuvée en 1996. Cette résolution complémentaire doit envisager une adéquation de la régulation principale aux spécificités des SHS. Cependant, il s'agit d'une ouverture de la CONEP qui ne répond pas aux revendications du Forum des SHS réuni en juillet 2013, étant donné que ce Forum envisage en fait la création d'un autre système d'évaluation éthique pour les SHS, indépendant de la CONEP et rattaché au Ministère de Sciences et Technologie, et non au Ministère de la santé.

Avec la participation de toutes les associations concernées, le Forum a discuté trois propositions de définition d'orientation éthique en vue d'un nouveau système d'éthique de la recherche. Chaque proposition a été discutée et une seule a été retenue. Ces trois propositions sont les suivantes : 1) l'exigence de « l'enregistrement universel » de tous les projets de recherche en SHS sans la nécessité d'une évaluation ; l'enregistrement aurait pour unique finalité de faciliter l'ouverture d'un processus en cas de soupçon d'une infraction au code d'éthique ; 2) la création d'un système «d'évaluation universel » de tous les projets de recherche en SHS ; 3) « enregistrement universel » de tous les projets mais " évaluations exceptionnelles » de quelques-uns. Grâce à une différenciation entre recherches portant un « risque minimal » et celles présentant un risque majeur, seules ces dernières seraient évaluées suite à une demande d'évaluation adressé au comité par le chercheur lui-même. La proposition qui a été retenue par le Forum de SHS est la plus conservatrice et bureaucratique des trois : la deuxième, l'évaluation universelle, qui présuppose la création d'un lourd dispositif institutionnel.

La dernière proposition se rapproche de la régulation homologue en vigueur au Canada et qui nous semble être très intéressante et novatrice. Le code d'éthique de recherche au Canada adopte le concept de risque minimal comme critère déterminant pour soumettre ou non une recherche à l'évaluation par un des trois comités d'éthique existants (qui sont : un pour les sciences médicales; un deuxième pour les sciences naturelles et le génie ; et un troisième pour les SHS). Un risque minimal est un risque qui ne se différencie pas de ceux auxquels le sujet est exposé au jour le jour dans sa vie quotidienne. Presque toutes les recherches en SHS se situent dans cette défini- 
tion-là et il se peut même qu'un projet de recherche clinique médicale soit dispensé d'évaluation s'il ne présente que des risques minimaux.

En résumé, au Brésil la régulation et la normalisation éthique en recherche sont très récentes, elles ont moins de deux décades d'existence ; cette régulation est strictement liée au champ de la santé visant prioritairement les interventions biomédicales; la pratique de recherche en SHS au Brésil est non seulement soumise à cette régulation biomédicale, mais manque encore d'une valorisation sociale et même académique par rapport aux sciences dites « dures».

Il faut souligner comment la transgression, surtout proposée par l'ABA et l'ANPOCS (Associação Nacional de Pós-graduação em Ciências Sociais) de l'éthique normative en vigueur a été fondamentale pour déclencher la révision du système officiel d'éthique en ce qui concerne les SHS. La situation des sciences humaines cliniques est plus délicate. Dès qu'il y a une intervention de ce type auprès de personnes, l'approbation par un comité d'éthique est exigée pour publier. Les juges de soutenance de thèses également commencent à refuser les travaux qui n'ont pas été soumis à un comité d'éthique. L'enjeu relatif à la publication joue un rôle de régulateur et de garde-fou devant la tentation de la transgression des lourdes procédures administratives. Se surajoute, en termes de complexité, le fait que sont en discussion au Congrès National des projets de loi qui pourront transformer les transgressions en crimes.

En France, contrairement au Brésil, le mouvement de généralisation de l'éthique procédurale n'a pas encore abouti. Il n'y a donc pas encore de totale institutionnalisation des instances d'éthique pour l'ensemble des disciplines de SHS. Contrairement aux revues médicales ou de psychologie, les revues de sciences sociales, sciences de l'éducation ou de sociologie n'exigent pas encore une approbation des comités d'éthique. Par conséquent, il demeure des dérives possibles surtout dans un tel contexte de pesanteur administrative. Ce sont donc les collectifs régulateurs de la recherche, en l'occurrence le financeur et quelques comités des revues scientifiques, qui jouent un rôle de garde-fou sur les questions éthiques.

\section{Quelques réflexions finales}

Au-delà des comités, on peut se demander quelle régulation de l'éthique est possible. Que ce soit en France ou au Brésil, on observe qu'une politique volontariste cherche à réguler éthiquement la recherche principalement dans le champ de la santé. Les gains en termes de protection ou au moins de tentatives de protection de la vie humaine sont évidents, spécialement dans un contexte d'expansion du « biopouvoir». Le cadrage légal contribue à faire face à la marchandisation de la santé, dont l'avancée démesurée de l'industrie pharmaceutique est la manifestation la plus évidente.

Au nom de la protection des personnes, il ne s'agit pas de positionner les chercheurs dans des situations inextricables, de les confronter à des logiques antagonistes. La confrontation à l'éthique procédurale amène à réfléchir à ce que l'on gagne en termes de responsabilisation accrue des chercheurs quand l'on diminue le poids du contrôle bureaucratique et que l'on augmente l'exposition au débat public de la recherche sociale. On gagne plus encore quand on inclut la participation active des sujets pendant le processus de recherche et non simplement au moment de la restitution de ses résultats - c'est ce que prétend la « recherche avec » ou participative. Mais il y a un prix à payer : ce type de recherche dépasse énormément le contrôle et la prévision envisagés par les procédures formelles. Le processus participatif implique une ouverture du parcours de recherche à des changements et à des incertitudes ; il demande une capacité du chercheur de mettre en réflexion théorique et éthique permanente ses strategies et ses rapports aux acteurs/sujets de recherche.

Les deux dispositifs centraux de la « recherche avec » sont la restitution et l'analyse de l'implication (LOURAU, 2004). La restitution veut dire que les résultats doivent être discutés avec les sujets intéressés avant d'être publiés ou diffusés, et l'analyse de l'implication signifie que le chercheur doit faire collectivement, avec les sujets et d'autres chercheurs, l'exercice d'explicitation de ses propres intérêts, valeurs et liaisons personnelles à son objet d'investigation et aux institutions (matérielles et symboliques) auxquelles il est rattaché. Au-delà de l'éthique procédurale, ces dispositifs peuvent devenir objets de considération de n'importe quelle recherche qualitative ou quantitative et non seulement de celles dites participatives. Tous les chercheurs devraient assumer le fait que leurs recherches produisent des effets sur la réalité investiguée, pas complètement connus d'avance ; qu'à travers elles, des éléments s'ajoutent à la réalité et par conséquent font partie de la réalité sociale. À moins de rester prisonnier d'une attitude positiviste naïve, qui suppose l'existence d'une réalité externe passible d'être absolument maîtrisée par le chercheur avec ses méthodes et ses instruments de recherche, il faut reconnaître que toutes les recherches ont une dimension socio-politique intrinsèque. Pour notre part, nous sommes convaincue que les résultats d'une recherche sont en quelque sorte les produits des instruments utilisés et du processus mis au jour. Comme l'explique Deleuze (2013), les évidences sont des choses qui se donnent à voir à partir de la vision et des instruments avec lesquels on voit ce qu'on voit. La tâche de la réflexion éthique est loin d'être accomplie par une éthique procédurale.

\section{Références}

ASSOCIATION FRANÇAISE DES ANTHROPOLOGUES. Éthique professionnelle et expérience de terrain . Journal des Anthropologues, n. 50-51, 1993.

ASSOCIATION MEDICALE MONDIALE. Déclaration d'Helsinki. Principes éthiques applicables à la recherche médicale impliquant des êtres humains. Helsinki : AMM, 1964. Disponible en : <http://www.wma.net/ fr/30publications/10policies/b3/>. Consulté le : 10 sept. 2014. 
BRASIL. Presidência da República. Casa Civil. Subchefia para Assuntos Jurídicos. Constituição da República Federativa do Brasil de 1988. Brasília, DF : Presidência da República, 1988. Disponível em : <http://www.planalto.gov.br/ccivil 03/ Constituicao/Constituicao.htm>. Acesso em : 22 nov. 2014.

BRASIL. Ministério da Saúde. Conselho Nacional de Saúde. Resolução $N^{o} 196$ de 10 de outubro de 1996. Diretrizes e normas regulamentadoras de pesquisas envolvendo seres humanos. Brasília, DF : Ministério da Saúde, 1996. Disponível em : <http://conselho.saude.gov.br/resolucoes/1996/Reso196. doc>. Acesso em : 22 nov. 2014.

BRASIL. Ministério da Saúde. Conselho Nacional de Saúde. Resolução $N^{o}$ 466, de 12 de dezembro de 2012. Brasília, DF: Ministério da Sáude, 2012. Disponível em : <http://conselho. saude.gov.br/resolucoes/2012/Reso466.pdf>. Acesso em : 10 set. 2014.

COMMISSION EUROPÉENNE. Charte européenne $d u$ chercheur: code de conduite pour le recrutement des chercheurs. Espace européen de la recherche : 2005. Disponible en : <http:// ec.europa.eu/euraxess/pdf/brochure_rights/eur_21620_en-fr. pdf>. Consulté le : 6 sept. 2014.

DELEUZE, G. El saber: curso sobre Foucault. Buenos Aires: Cactus, 2013.t. 1.

DIET, É. L'homme procédural. De la perversion sociale à la désubjectivation aliénante. Connexions, v. 1, n. 79, p. 1128, 2003. Disponible en : <http://www.cairn.info/revueconnexions-2003-1-page-11.htm>. Consulté le : 12 sept. 2014.

DINIZ, D. Ética na pesquisa em ciências humanas - novos desafios. Ciência \& saúde coletiva, v. 13, n. 2, p. 417-426, 2008.

FASSIN, D. L'éthique, au-delà de la règle : réflexions autour d'une enquête ethnographique sur les pratiques de soins en Afrique du Sud. Sociétés contemporaines, v. 3, n. 71, p. 117$135,2008$.

FRANCE. Loi $n^{\circ} 88-1138$ du 20 décembre 1988 relative à la protection des personnes qui se prêtent à des recherches biomédicales « Loi Huriet-Sérusclat ». 1988. Disponible en : <http://legifrance.gouv.fr/affichTexte. do? cidTexte=JORFTEXT000000508831>. Consulté le : 5 juil. 2014

FRANCE.Loin ${ }^{\circ}$ 2004-806du9août2004relativeàlapolitiquede santé publique. 2004a. Disponible en $:<$ http://www.legifrance. gouv.fr/affichTexte.do? cidTexte=JORFTEXT000000787078>. Consulté le : 6 sept. 2014.

FRANCE. Article $n^{\circ} 90$ Code de la Santé publique. Modifié par la loi No. 2004-806 (2004, 9 aôut) relative à la politique de santé publique. 2004b. Disponible en : <http://www.legifrance. gouv.fr>. Consulté le : 6 sept. 2014.

FRANCE. Loi $n^{o}$ 2009-879 du 21 juillet 2009 portant réforme de l'hôpital et relative aux patients, à la santé et aux territoires « Loi Hôpital, patients, santé et territoires ». 2009. Disponible en : <http://www.legifrance.gouv.fr/affichTexte. do? cidTexte $=$ JORFTEXT000020879475\&categorieLien $=i d>$. Consulté le : 6 sept. 2014.

FRANCE. Loi $n^{\circ}$ 2012-300 du 5 mars 2012 relative aux recherches impliquant la personne humaine « Loi Jardé ». 2012. Disponible en : <http://www.legifrance.gouv.fr>. Consulté le : 6 sept. 2014.
GARRAFA, V.; LORENZO, C. As constantes mudanças na Declaração de Helsinque: redução de proteção e maximização de interesses privados. In: CAPONI, S. et al. (Org.). Medicalização da vida: ética, saúde pública e indústria farmacêutica. 2. ed. Curitiba: Prismas, 2013. p.19-36.

GUERRIERO, I. C. Z. Aspectos éticos das pesquisas qualitativas em saúde. 2006. Tese (Doutorado)-Faculdade de Saúde Pública, Universidade de São Paulo, São Paulo, 2006.

HARDY, A.-C. Travailler à guérir: sociologie de l'objet du travail médical. Rennes: EHESP, 2013.

KATZ, J. Ethical escape routes for underground ethnographers. American Ethnologist, v. 33, n. 4, p. 499-506, 2006.

LANGDON, E. J. Dilemas da pesquisa qualitativa frente à legislação sobre ética: o relatório do encontro "Ética em Pesquisa Qualitativa em Saúde”. In : CARDOSO, V. Z. (Org.). Diálogos Transversais em Antropologia. Florianópolis, SC: UFSC, 2008. p. 127-135.

LAURENS, S.; NEYRAT, F. Enquêter : de quel droit? Menaces sur l'enquête en sciences sociales. Paris : Croquant, 2010.

LOURAU, R. Implicação e sobreimplicação. In: ALTOÉ, S. (Org.). René Lourau analista institucional em tempo integral. Tradução de A. P. J. Melo. São Paulo: Hucitec, 2004. p. 186198. Original publicado Revue du Mauss, v. 10, n. 4, 1990.

MARTINEZ-HERNAEZ, A. A medicalização dos estados de ânimo: o consumo de antidepressivos e as novas biopolíticas das aflições. In: CAPONI, S. et al. (Org.). Medicalização da vida: ética, saúde pública e indústria farmacêutica. 2. ed. Curitiba: Prismas, 2013. p.95-114.

MINAYO, M. C. S. Contribuições da antropologia para dilemas éticos da área da saúde. Ciênc. saúde coletiva (eletrônica), v. 13, n. 2, p. 329-339, 2008. Disponível em: <http://dx.doi. org/10.1590/S1413-81232008000200008>. Acesso em : 15 set. 2014.

ORGANISATION MONDIALE DE LA SANTÉ; CONSEIL DES ORGANISATIONS INTERNATIONALES DES SCIENCES MÉDICALES. Déclaration de Manille. Directives internationales proposées pour la recherche biomédicale impliquant des sujets humains. Manile, OMS/ CIOMS, 1981. Disponible en : <http://www.tbethics.org/Textes/Declaration de_Manille-VF.pdf >. Consulté le : 10 sept. 2014.

PARLEMENT EUROPÉEN. Directive européenne 2001/20/CE. Parlement européen et conseil concernant le rapprochement des dispositions législatives, règlementaires et administratives des États membres, relatives à l'application de bonnes pratiques cliniques dans la conduite d'essais cliniques de médicaments à usage humain. 4 avril 2001. Disponible en : <http://eur-lex.europa.eu/LexUriServ/LexUriServ. do?uri=OJ:L:2001:121:0034:0044:fr:PDF>. Consulté le : 6 sept. 2014.

RED LATINOAMERICANA Y DEL CARIBE DE BIOETICA / UNESCO. Carta de Córdoba sobre ética en investigaciones con seres humanos. 2008. Disponible en : <http://www.psi. uba.ar/academica/carrerasdegrado/psicologia/sitios_catedras/ obligatorias/723_etica $2 /$ material/casuistica/carta_de_ cordoba_08.pdf>. Consulté le : 12 janv. 2015.

SARTI, C.; DUARTE, L. F. D. (Org.). Antropologia e ética: desafios para a regulamentação. Brasília, DF: ABA, 2013. 
SCHLEMPER JUNIOR, B. ; HELLMANN, F. Controvérsias em tempos de mudanças na Declaração de Helsinque e a experiência brasileira em ética em pesquisa. In : CAPONI, S. et al. (Org.). Medicalização da vida: ética, saúde pública e indústria farmacêutica. 2. ed. Curitiba: Prismas, 2013. p. 37-66.

UNITED STATES. United States Department of Health, Education, and Welfare. Rapport Belmont. 1979. Principes éthiques et lignes de conduites pour la protection des sujets humains de la recherche. États-Unis : Département de la santé, de l'éducation et des services sociaux des États-Unis. (Trad.) 1979. In: HARMS, D. Ethical principles and guidelines for the protection of human subjects of research: Belmont report. United States: HEW, 1979.

VASSY, C.; KELLER, R. Faut-il contrôler les aspects éthiques de la recherche en sciences sociales, et comment? Mouvements, v. 3, n. 55-56, p. 128-141, 2008. Disponible en : <www.cairn. info/revue-mouvements-2008-3-page-128.htm>. Consulté le : 10 sept. 2014.

Reçu: Février 1, 2015

Accepté: Juillet 5, 2015 